\title{
THE CROSSROADS OF COGNITIVE SCIENCE
}

The monograph Cognition, Meaning and Action. Lodz-Lund Studies in Cognitive Science collects papers written by the members of two Cognitive Science Departments: of Lund and of Lodz. It presents a range of issues currently examined in both centers. Some texts are written in collaboration as the result of collective research.

The opening article "Cognitive science: From computers to ant hills as models of human thought" (Peter Gärdenfors) offers an introduction to the history of ideas in cognitive science as it has been developing throughout last decades. Much of the contemporary mind theories derive from Descartes' res cogitans and res extensa distinction, and to some extent they may be seen as a continuation of rationalist-empiricist debate. The dawn of computer science is kept in quite rationalist fashion. The fundamental concept of computer science is the theoretical construct of Turing's machine. Inspired by Turing's concept, John von Neumann proposes a general architecture for modern computer based on logic circuits. The transfer of these findings to a theory of how the mind works was only a matter of time. Soon after von Neumann's proposal, McCulloch and Pitts interpreted neurons as a logic circuits combining information from other neurons according to some logical operations. This leads directly to one conclusion: the entire brain is a huge computer - and so the foundational metaphor for cognitive science was born.

Cognitive science can be said to emerge in 1956, the year in which Noam Chomsky, in response to the behaviourist concept of language, presented his proposition of transformational grammar. His central argument is based on the claim that processing the grammar of natural language requires a sort of algorithm as used in Turing machine. Also in 1956 Newell and Simon demonstrated the first computer program constructing logical proofs from a given set of premises 
and, finally, the concept of Artificial Intelligence was used for the first time. The philosophical assumption of the AI approach to cognitive processes is that the representation of mental content and processing is essentially symbol manipulation: only logical relations connect different symbolic expressions in a mental state of a person. The meaning of symbols is not part of the process of thinking, since they are manipulated exclusively on the basis of their form.

This quite rationalist manner of representing the cognitive process gave rise to several forms of criticism. One of them - derived from empiricism - was a new model of cognition called connectionism. Connectionist systems, also called artificial neuron networks, consist of large number of simple but highly interconnected units ("neurons"). According to the connectionists' point of view, thinking is not manipulation of meaningless symbols run and controlled by a central processor computer-like program, but it rather occurs in parallel neuronal processes distributed all over the brain, which is seen as a self-organizing system.

However, as it is claimed in the first paper, there are aspects of cognitive phenomena for which neither symbolic representation nor connectionism seems to offer appropriate "modelling tools". Those aspects include: mechanisms of concept acquisition, concept learning, and the notion of similarity. They turned out to be problematic for the symbolic and associationist approaches. To deal with them, a third form of representing information was proposed based not on symbols or connections between neurons, but rather on geometrical or topological structures. These structures generate mental spaces that represent various domains, and allow for modelling similarity in a very natural way as, for example, with the function of distance in such a space.

The topics of all other papers oscillate around the eponymous subject from the point of view of communication and its efficiency. The philosophical perspective of thinking, typical for the research on cognition, meaning, and action, is here replaced by psychological as well as neurophysiological benchmarks. The concept of the meaning of natural language expressions presented in "Two procedures expanding a linguistic competence" (Piotr Eukowski) is the result of two approaches, of the logical and of the one known in the cognitive psychology as exemplary theory of meaning. It employs model example, function of sufficient similarity, accidental and essential similarities and zone of proximal development. From such a perspective, the meaning inevitably appears to be a social, dynamic, and temporal phenomenon. Furthermore, since cognitive psychology is firmly founded on neuroscientific research, the properties of the presented understand- 
ing of notions can be partially linked to their neurophysiological correlates, as outlined in the following chapter: "Neurobiological basis for emergence of notions" (Konrad Rudnicki).

Comparative studies of feature lists, (dynamic) frames, and conceptual spaces as models for the representation of scientific conceptual knowledge is the aim of "Similarity as distance: Three models for scientific conceptual knowledge" (Frank Zenker). It is shown that the concepts arising from and giving rise to the exact measurement - mainly scientific ones - are properly represented in conceptual spaces. Also in the paper "The Approximate Numbers System and the treatment of vagueness in conceptual spaces" (Aleksander Gemel, Paula Quinon) the advantages of this model are successfully confirmed for the representation of concepts whose character is far from being scientific, i.e. vague concept of number.

Interpersonal communication defines the context of analyses for the next two papers: "To tell and to show: the interplay of language and visualizations in communication" (Jana Holsanova, Roger Johansson, Kenneth Holmqvist) and "Communication, cognition, and technology" (Peter Gärdenfors, Jana Holsanova). The main topic of both texts concerns various kinds of visualization with particular focus on how they influence communicational effectiveness. Structuralist semiotics and naturalistic, computational concepts of language are traditionally considered as being in conflict. Yet, closer analysis reveals their complementarity. In the paper "Semiotics, signaling games and meaning" (Aleksander Gemel, Bartosz Żukowski) some reconciliation of these two paradigms is proposed, which results in a coherent model preserving the advantages of the both concepts. The hybrid model requires, however, a formal tool to organize the semantic structure of the cultural system. To this aim content implication is introduced.

Starting from the following paper, rational action is the leading problem for all texts. The first of them, "Out of the box thinking" (Dorota Rybarkiewicz) explains in terms of the theory of metaphor how to break natural, standard borders - our typical canyons of thought - in order to find a better solution of a given problem. Procedures of decision making are analyzed in two papers closing the volume: "The everyday of decision-making" (Annika Wallin) and "Short- and long-term social interactions from the game theoretical perspective: A cognitive approach” (Magdalena Grothe, Bartosz Żukowski). In the former, the study of human everyday practice becomes the source of truths (information) about what a real and rational decision process looks like and of ideas about how to improve this process. In the latter, the rationality of decision making is steeped in 
the game theory. The well-known results established for the models of prisoner's dilemma and those with an indefinite time framework are related to the social interactions which are consistent with the cooperative equilibrium over a longer time.

Peter Gärdenfors (Department of Cognitive Science, Lund)

Piotr Łukowski (Department of Cognitive Science, Łódź) Łódź, March 2015 